\title{
INVESTIGATING THE POTENTIAL TO ESTIMATE INSAR PENETRATION DEPTH OVER ICE SHEETS FROM POL-INSAR DATA
}

\author{
Georg Fischer ${ }^{1,2}$ (georg.fischer@dlr.de), Giuseppe Parrella ${ }^{1}$, Konstantinos P. Papathanassiou ${ }^{l}$, Irena \\ Hajnsek $^{1,2}$
}

${ }^{1}$ DLR, Microwaves and Radar Institute, Germany; ${ }^{2}$ ETH Zurich, Institute of Environmental Engineering

\begin{abstract}
Digital elevation models generated with SAR interferometry (InSAR) are an important information source for glacier and ice sheet mass balance. However, the measured elevations suffer from a penetration bias due to the interferometric phase center being up to several tens of meters below the surface. The penetration of the microwave signals depends on SAR parameters (e.g. frequency) and snow and ice conditions. There is potential to estimate this penetration bias directly from the data by means of polarimetric InSAR models. Existing models fail to describe the data across different test sites and ice conditions and phase centers were found to be deeper than predicted by these models. SAR tomography is employed to assess the vertical distribution of backscattering in the data from an airborne campaign. The data are compared to refined models in order to find better representations of the vertical backscattering distribution, while the model complexity is purposely kept simple to make a phase center estimation possible. Additionally, recent work showed the importance of strong subsurface layers which influence phase center depth. Combining the refined subsurface structure models and dominant subsurface layers allows simulating a variety of ice sheet subsurface scenarios and can be used to assess the potential to estimate the InSAR phase center depth directly from the data.
\end{abstract}

Index Terms - Pol-InSAR, phase center, penetration bias, subsurface, glaciers

\section{INTRODUCTION}

The estimation of the mass balance of glaciers and ice sheets is important for assessing their temporal and spatial dynamics [1]. Digital elevation models (DEMs) generated with synthetic aperture radar interferometry (InSAR) [2] provide valuable information for mass balance estimates due to their large coverage, high spatial resolution and all-day, weather independent acquisitions. However, the penetration of the microwave signals into snow and ice under dry and frozen conditions causes an inherent bias in InSAR DEMs acquired over glaciers and ice sheets, which can be on the order of tens of meters for systems with longer wavelengths, e.g. at L-band [3]. There is potential to estimate and mitigate this penetration bias directly from the data. Studies have shown the retrieval of InSAR penetration depths [4] and signal extinction coefficients [5] at different frequencies and polarizations based on the assumption of a lossy signal propagation through an isotropic, homogeneous volume in the subsurface of glaciers or ice sheets, which is referred to as Uniform Volume (UV) model. However, InSAR phase centers have been found to be deeper than predicted by the UV model [6][7].

This indicates the necessity of refined InSAR models for the vertical distribution of backscattering in the subsurface of glaciers and ice sheets. Results from SAR tomography show a more heterogeneous vertical backscattering distribution at two test sites in the percolation zone of the Greenland ice sheet and highlight the presence of surface-like scattering layers in the subsurface. Accordingly, non-uniform volume models and Dirac deltas are used to simulate the data. Based on the simulations and the data, it is the objective of this study to investigate how accurate the InSAR penetration bias can be estimated based on different model assumptions, constrained by the tradeoff between observation space and modelling complexity.

\section{TEST SITES AND SAR DATA}

The SAR data used in this study were acquired in April and May 2015 in Greenland during the airborne ARCTIC15 campaign of DLR and ETH Zurich. The two presented test sites, located in the percolation zone, are South Dome $\left(63.52^{\circ} \mathrm{N}, 44.54^{\circ} \mathrm{W}\right.$, Alt.: 

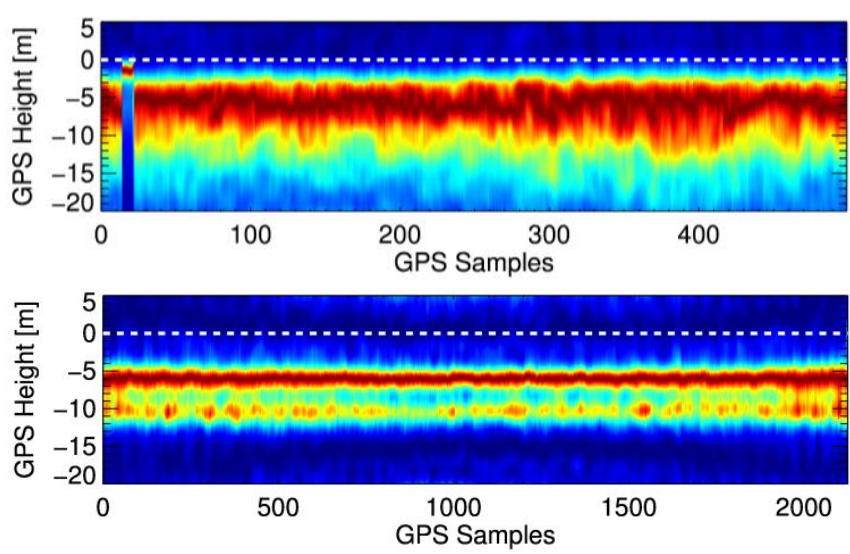

Fig. 1. Tomographic profile derived with the Capon adaptive beamformer from L-band VV data at EGIG T05 (top) and L-band VV data at South Dome (bottom). The effect of a corner reflector is visible at the EGIG T05 profile.

$2868 \mathrm{~m})$ and EGIG T05 $\left(69.87^{\circ} \mathrm{N}, 47.13^{\circ} \mathrm{W}\right.$, Alt.: $1938 \mathrm{~m}$ ). The melting period in summer is longer and more intense at EGIG T05 due to its lower elevation. Accordingly, an abundance of ice inclusions, originating from refrozen melt water, can be assumed within the firn body. This is confirmed by the tomographic profile shown in Fig. 1 (top), where continuous strong scattering can be found below the surface, likely originating from the ice inclusions. On the contrary, the shorter and weaker melt period at South Dome causes fewer inclusions which are limited in their vertical distribution, resulting in distinct seasonal layers, see Fig. 1 (bottom). The tomograms are referenced to surface GNSS measurements and the general subsurface scattering structure is confirmed by ground penetrating radar data, both acquired during the ARCTIC15 campaign [6]. The first few meters below the surface consist of winter snow and young firn with less refrozen ice inclusions and are mainly transparent.

\section{INSAR PHASE CENTER DEPTHS}

InSAR phase center depths, shown in Fig. 2, are derived at the same locations as the tomograms in Fig. 1 and are referenced to the surface GNSS measurements. Phase centers are shown for $\mathrm{HH}, \mathrm{VV}$ and HV polarization at L-band at EGIG T05, Fig. 2 (top) and South Dome, Fig. 2 (bottom). The data are from interferograms acquired with different baselines which provide a range of vertical wavenumbers $k_{Z V o l}$. The vertical wavenumber $k_{z V o l}$ describes the sensitivity of interferometric phase to height considering refraction and permittivity in the subsurface [5]
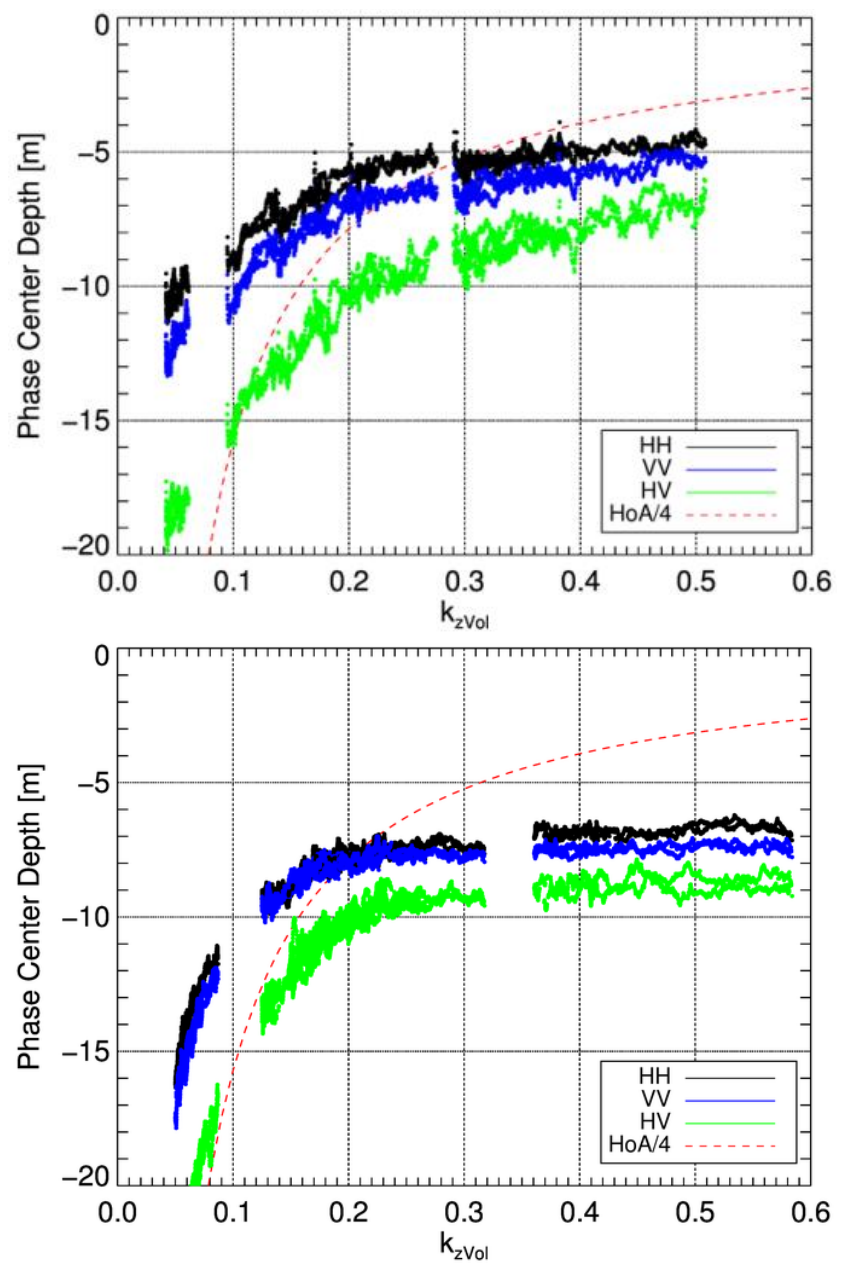

Fig. 2. InSAR phase center depth referenced to GNSS measurements at the surface over a range of vertical wavenumbers $k_{Z V o l}$ at L-band in $\mathrm{HH}, \mathrm{VV}$ and $\mathrm{HV}$ polarizations at EGIG T05 (top) and South Dome (bottom). The red dashed line indicates the lower limit of a UV model.

$$
k_{z V o l}=\frac{4 \pi \sqrt{\epsilon_{r}}}{\lambda} \frac{\Delta \theta_{r}}{\sin \theta_{r}} .
$$

Here, $\lambda$ is the wavelength in free space, $\epsilon_{r}$ the bulk relative permittivity of the subsurface in which the signal penetrates into and $\Delta \theta_{r}$ is the angular difference between the two radar look vectors of the interferograms, driven by the baseline between the two acquisition tracks, after considering refraction into the firn volume.

Under the assumption of a constant signal extinction (i.e. an exponential vertical backscattering distribution), the lower limit of the UV model is a quarter of the InSAR height of ambiguity [8], which is inversely related to $k_{z V o l}$. This lower limit is indicated in Fig. 2 by the red dashed line and the measured 
phase centers are below this limit. This, together with the tomograms, demonstrates the need for refined InSAR models in order to estimate phase center depths.

\section{SUBSURFACE STRUCTURE MODELLING}

InSAR phase centers are extracted from interferometric coherences, which can be modelled after common bandwidth filtering, neglecting temporal and noise decorrelation, as

$$
\gamma=e^{i k_{z} z_{0}} \frac{\int_{-\infty}^{0} \sigma_{v}(z) e^{i k_{z V o l} z} d z}{\int_{-\infty}^{0} \sigma_{v}(z) d z}
$$

The coherence depends on the vertical backscattering distribution $\sigma_{v}(z)$, stretching from $z_{0}$ at the glacier surface to depth $z$. Additionally, dominant subsurface layers need to be considered, as indicated by the tomogram in Fig. 1 (bottom). These $N$ layers can be simulated by Dirac delta functions located at depth $z_{j}$ with layer-to-volume scattering ratio $m_{j}$ leading to

$$
\gamma=e^{i k_{z} z_{0}} \frac{\gamma_{V o l}\left(k_{Z V o l}, d_{p e n}\right)+\sum_{j=1}^{N} m_{j} e^{i k_{Z V o l} z_{j}}}{1+\sum_{j=1}^{N} m_{j}}
$$

with $\gamma_{V o l}\left(k_{z V o l}, d_{p e n}\right)$ being the output of a volume model.

Three volume models have been tested against the tomographic data (Fig. 1) in order to find a better model representation of the vertical backscattering structure to improve phase center estimation. First, a UV model with constant extinction [4] but an additional vertical shift, to account for the first few transparent meters below the surface in the tomograms. Second, a Gaussian function with mean depth and std. dev. of the vertical backscattering distribution. And third a Weibull function, which is fixed to start at the surface, but has a shape parameter $k$ which allows the transition from a UV model (see $k=1$ in Fig. 3) to a Gaussian (see $k=2.5$ in Fig. 3).

Calculating a best-fit between the tomograms from the data and the volume models showed that the UV model with a vertical shift fits best to the EGIG T05 data, while the Gaussian and Weibull models perform better for the South Dome data. Note that the two strong scattering layers in the South Dome data were attenuated [9] before calculating the best-fit. All three

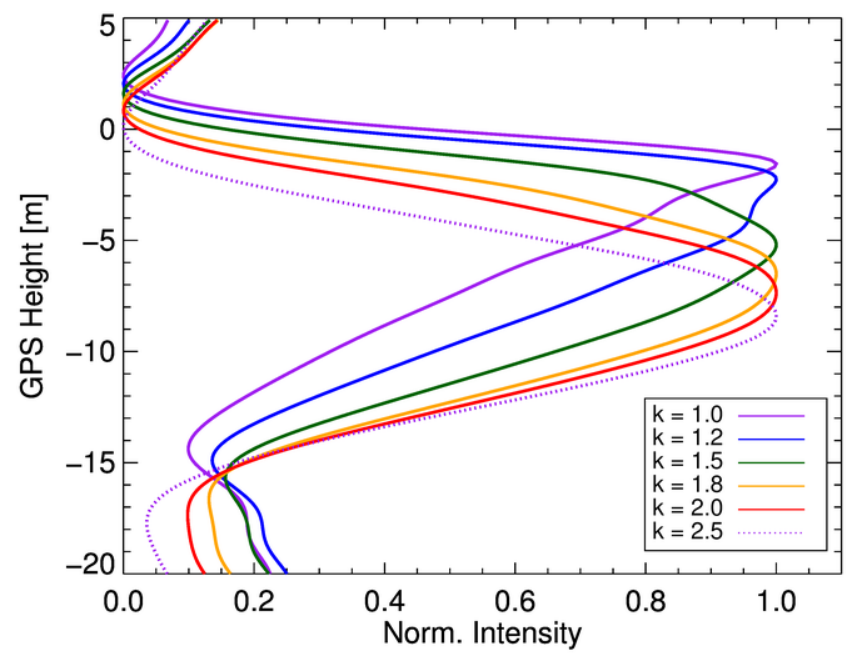

Fig. 3. Capon response of Weibull model with variable shape parameter $k$ and fixed scale parameter $\lambda_{w}=0.10$ with South Dome L-band imaging geometry.

tested models performed significantly better than the UV model [4] with a fixed upper limit at $z=0$.

\section{CONCLUSIONS AND OUTLOOK TOWARDS PHASE CENTER ESTIMATION}

The models introduced in Section 4 provide more flexibility to simulate vertical backscattering distributions in ice sheets. A shifted UV model can represent the EGIG T05 data, while the South Dome case can be simulated with a Weibull or Gaussian function for the volume and two Dirac deltas for the dominant subsurface layers. This provides a test bed for phase center estimation approaches. Potential lies in retrieval techniques with polarimetric InSAR [10], where polarimetry can support to separate the effects of layers and volume. The observation space can be further increased by using more than one baseline, where recent studies showed that small baselines provide more sensitivity to the volume and larger baselines more sensitivity to subsurface layers [11].

For the phase center depth estimation, it is unknown beforehand which volume model represents the data the best. In addition it is unclear, if the volume model is similar for each polarization and further, if and how many additional subsurface layers have to be considered.

This study investigates which volume model performs best for the phase center estimation from different simulation scenarios and the presented data. In this context, it is important if the same volume can be assumed for all polarizations (random volume). 


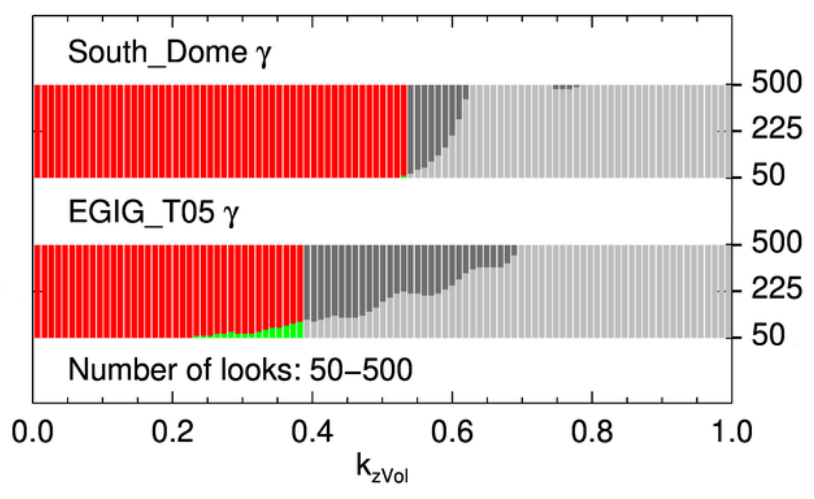

Fig. 4. The plot indicates if the complex coherences derived for $0<k_{z V o l}<1$ from the tomographic profiles at $\mathrm{HH}, \mathrm{VV}$ and HV polarizations are within each other's standard deviation (green) or not (red). This is done for both test sites and for 50 to 500 looks and is used as an indicator for the validity of the random volume assumption. Red and green are changed to dark and light gray, respectively, when the coherence magnitude of all three polarizations drops below 0.2 .

This can be assessed, for instance, by calculating InSAR coherences from the tomographic profiles at both test sites across a range of $k_{Z \mathrm{VOl}}$ and number of looks for $\mathrm{HH}, \mathrm{VV}$ and $\mathrm{HV}$ polarizations and then testing if the coherences from the three polarizations are within each others standard deviation, see Fig. 4. First results indicate that the assumption does not hold, but it will be investigated how strongly the random volume assumption affects the phase center estimation, because it simplifies the estimation procedure and reduces the requirements on the observation space.

A further question is if the number and intensity of subsurface layers can be estimated or if their effect on the phase center can be approximated by the volume models alone, which would also reduce the requirements on the observation space.

The results will finally indicate if the InSAR penetration bias in DEMs over glaciers and ice sheets can be estimated and mitigated directly from the data of polarimetric and interferometric SAR missions, which is particularly relevant at longer wavelengths, e.g. L-band.

\section{REFERENCES}

[1] D.G. Vaughan, J.C. Comiso, I. Allison, J. Carrasco, G. Kaser, R. Kwok, P. Mote, T. Murray, F. Paul, J. Ren, E. Rignot, O. Solomina, K. Steffen, and T. Zhang, "Observations: Cryosphere," in Climate Change 2013: The Physical Science Basis. Contribution of Working Group I to the Fifth Assessment Report of the Intergovernmental Panel on Climate Change, [Stocker, T.F., D. Qin, G.-K. Plattner, M. Tignor, S.K. Allen, J. Boschung, A. Nauels, Y. Xia, V. Bex and P.M. Midgley (eds.)]. Cambridge University Press, Cambridge, United Kingdom and New York, USA, 2013.

[2] B. Wessel, A. Bertram, A. Gruber, S. Bemm, and S. Dech, "A New High-Resolution Elevation Model of Greenland Derived from Tandem-X," ISPRS Annals of Photogrammetry, Remote Sensing and Spatial Information Sciences, vol. III-7, pp. 916, Jun. 2016.

[3] E. Rignot, K. Echelmeyer, and W. Krabill, "Penetration depth of interferometric synthetic aperture radar signals in snow and ice," Geophys. Res. Lett., vol. 28, no. 18, pp. 3501-3504, Sep. 2001.

[4] E. W. Hoen and H. Zebker, "Penetration depths inferred from interferometric volume decorrelation observed over the Greenland ice sheet," IEEE Trans. Geosci. Remote Sens., vol. 38, no. 6, pp. 2572-2583, Nov. 2000.

[5] J.J. Sharma, I. Hajnsek, and K.P. Papathanassiou, "Estimation of glacier ice extinction using long-wavelength airborne PolInSAR," IEEE Trans. Geosci. Remote Sens., vol. 51, no. 6, pp. 3715-3732, Jun. 2013.

[6] G. Fischer, G. Parrella, K. P. Papathanassiou, and I. Hajnsek, "Sensitivity of polarimetric SAR interferometry data to different vertical subsurface structures of the Greenland ice sheet," in Proc. of IGARSS, Forth Worth, USA, 2017, pp. 3581-3584.

[7] J. Dall, K.P. Papathanassiou, and H. Skriver, "Polarimetric SAR interferometry applied to land ice: Modeling," in Proc. EUSAR, Ulm, Germany, 2004, pp. 247-250.

[8] J. Dall, "InSAR Elevation Bias Caused by Penetration Into Uniform Volumes," IEEE Transactions on Geoscience and Remote Sensing, vol. 45, no. 7, pp. 2319-2324, Jul. 2007.

[9] G. Fischer, M. Pardini, G. Parrella, K. P. Papathanassiou, and I. Hajnsek, "Cancellation of Dominant Scattering Layers in Greenland's Subsurface for the Characterization of Ice Volumes," in Proc. EUSAR, Aachen, Germany, Jun. 4-7, 2018.

[10] S.R. Cloude and K.P. Papathanassiou, "Polarimetric SAR interferometry," IEEE Trans. Geosci. Remote Sens., vol. 36 , no. 5, pp. 1551-1565, Sept. 1998.

[11] G. Fischer, K. P. Papathanassiou, and I. Hajnsek, "Modeling Multifrequency Pol-InSAR Data From the Percolation Zone of the Greenland Ice Sheet," IEEE Transactions on Geoscience and Remote Sensing, pp. 1-14, Early Access, 2018. 